\title{
Power Flow in Four-Wire Distribution Networks - General Approach
}

\author{
Rade M. Ciric, Antonio Padilha, Member, IEEE, and Luis F. Ochoa, Student Member, IEEE
}

\begin{abstract}
The neutral wire in most power flow software is usually merged into phase wires using Kron's reduction. Since the neutral wire and the ground are not explicitly represented, neutral wire and ground currents and voltages remain unknown. In some applications, like power quality and safety analyses, loss analysis, etc., knowing the neutral wire and ground currents and voltages could be of special interest. In this paper, a general power flow algorithm for three-phase four-wire radial distribution networks, considering neutral grounding, based on backward-forward technique, is proposed. In this novel use of the technique, both the neutral wire and ground are explicitly represented. A problem of three-phase distribution system with earth return, as a special case of a four-wire network, is also elucidated. Results obtained from several case studies using medium and low voltage test feeders with unbalanced load, are presented and discussed.
\end{abstract}

Index Terms-- Distribution networks, power flow, three-phase four-wire, earth return, unbalance.

\section{INTRODUCTION}

$\mathrm{T}$ HREE-phase four-wire distribution networks (DNs) are widely adopted in modern power distribution systems [1], [2], [3], [4] and [5]. A multi-grounded three-phase four-wire service has higher sensitivity for fault protection than a threephase three-wire service. The return current is due to both the unbalanced load and the non-linear characteristics of electrical equipment through the distribution feeder. The return current may be larger than the phase currents if three-phase loads are seriously unbalanced in some segments. The neutrals play an important role in power quality and safety problems [6], [7] and [8]. The design of neutrals varies widely from country to country and even from utility to utility within the same country. The different technical solutions considering the neutral in medium voltage are: systems without neutral (Brazil), systems with an isolated neutral (Italy, Finland, Switzerland), systems with multiple grounding of the neutral and connecting the utility neutral with customer grounding (USA, Greece), systems with solid neutral grounding (UK), systems with resistance neutral grounding (France, UK), systems with reactance neutral grounding (Belgium, Spain, Portugal, Netherlands), and systems compensated via a

The authors would like to thank the ELUCID Solutions S.A., Sao Paulo, SP, Brazil, and FAPESP (contract no. 00/120099-1), for their financial support and commitment to this project.

R. M. Ciric is presently a postdoctoral researcher at UNESP - Ilha Solteira, SP, Brazil.

A. Padilha is with UNESP - Ilha Solteira, SP, Brazil.

L. F. Ochoa is a M.Sc. Student at UNESP - Ilha Solteira, SP, Brazil.
"Petersen" coil (Germany), to name a few [3].

An efficient and robust three-phase power flow algorithm for large-scale DNs with dispersed generators is the chief tool for a Distribution Management System (DMS) today. In the last decade, different procedures have been proposed for distribution power flow [9], [10], [11], [12], [13], [14] and [15]. Experience demonstrates that very good results are obtained using the backward-forward procedure while handling large-scale real-life DNs. These methods may be classified as current summation methods, power summation methods and admittance summation methods.

The neutral wire in the existing power flow software is usually merged into phase wires using Kron's reduction. Since the neutral wire and ground are not explicitly represented, neutral wire and ground currents and voltages remain unknown. In some applications like power quality and safety analyses, loss analysis etc., it is of special interest to ascertain the neutral wire and ground currents and voltages.

In order to calculate neutral wire and ground currents and voltages, here the efficient and robust three-phase branchoriented backward-forward procedure [10] is generalized. It is expanded the $3 \times 3$ network representation to a $5 \times 5$ representation, considering three-phase wires, neutral wire and assumed ground wire. The developed distribution power flow method is of general usage since it can be applied for most of the existing DNs: medium voltage (MV); low voltage (LV); three-wire, four-wire, with (solidly) grounded neutral, or isolated neutral wire; and three-wire and single-wire DNs with earth return. A problem of a three-phase distribution system with an earth return distribution [16] is also elucidated by the employment of the proposed generalized power flow algorithm, as a special case of a four-wire network, which can be found in Brazil, South Africa, Iceland and Australia, among others.

The main objectives of this work are to demonstrate that higher order line models can be included in the procedure of [10] with minor changes and then to determine the effect of this inclusion on the accuracy of the solution.

\section{POWER Flow AlgorithM}

In the three-phase power flow algorithm, each node or line section in the network is numbered by a single index, regardless of the number of phases of this node or line section [10]. Figure 1 represents line section $l$ between nodes $i$ and $j$ with shunt admittances and loads attached to the nodes. 


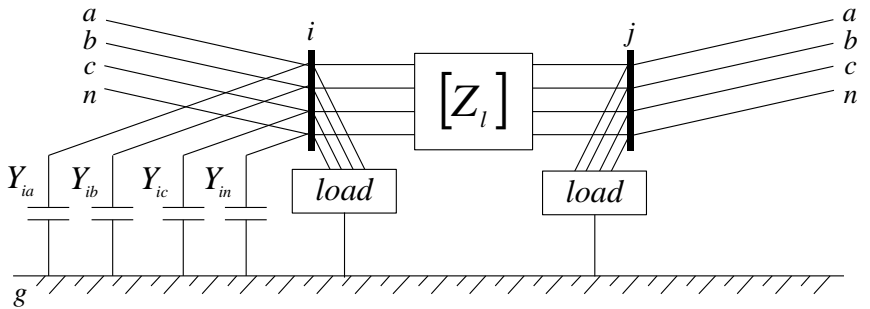

Fig. 1. A three-phase four-wire line section, considering ground.

Based on Fig. 1, a $5 \times 5$ matrix can represent the series impedance $Z_{l}$ of the line section $l$ :

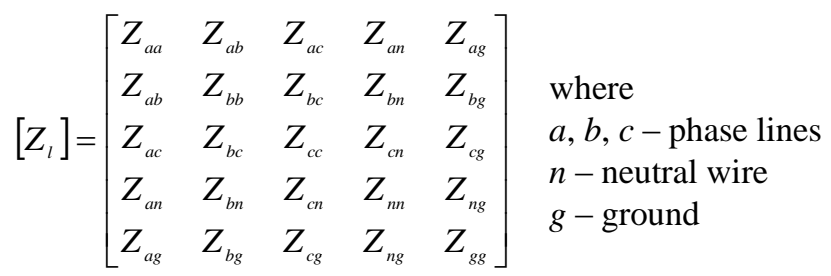

If any phase, neutral wire or grounding of the line section does not exist, the corresponding row and column in this matrix contain all zero entries. The shunt capacitance part of $\mathrm{LV}$ lines can be neglected. Branch numbering scheme for radial DN is exactly like in [10].

\section{A. Model}

Model of the three-phase four-wire multi-grounded distribution line is shown on Fig. 2.

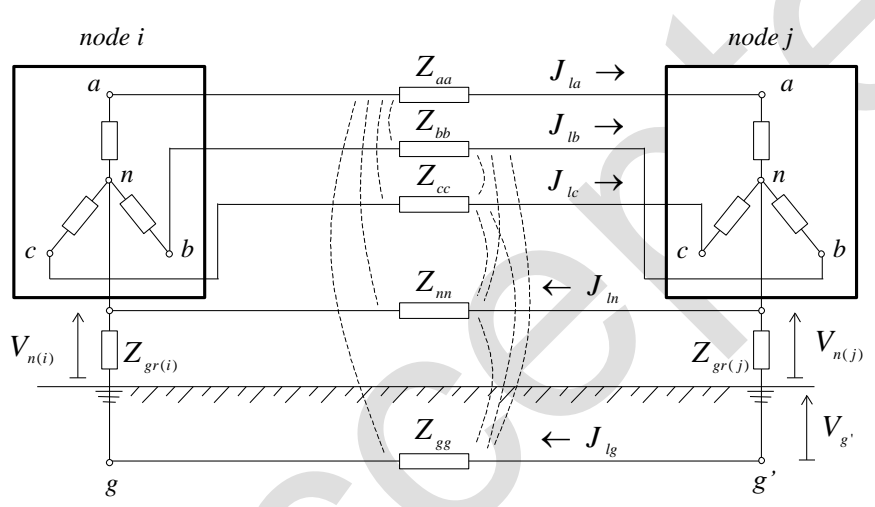

Fig. 2. Model of the three-phase four-wire multi-grounded distribution line.

\section{B. Power Flow Algorithm}

Assuming the root node to be the slack node with known voltage magnitude and angle, here the procedure from [10] is generalized. The proposed $5 \times 5$ iterative algorithm to solve the radial system consists of three steps, as follows.

At iteration $k$ :

1. Nodal current calculation

$$
\left[\begin{array}{c}
I_{i a} \\
I_{i b} \\
I_{i c} \\
I_{i n} \\
I_{i g}
\end{array}\right]^{(k)}=\left[\begin{array}{c}
\left(S_{i a} / V_{i a}\right)^{(k-1)^{*}} \\
\left(S_{i b} / V_{i b}\right)^{(k-1)^{*}} \\
\left(S_{i c} / V_{i c}\right)^{(k-1)^{*}} \\
-\frac{Z_{g i}}{Z_{n n i}+Z_{g i}}\left(I_{i a}^{(k)}+I_{i b}^{(k)}+I_{i c}^{(k)}\right) \\
-\frac{Z_{n n i}}{Z_{n n i}+Z_{g i}}\left(I_{i a}^{(k)}+I_{i b}^{(k)}+I_{i c}^{(k)}\right)
\end{array}\right]
$$

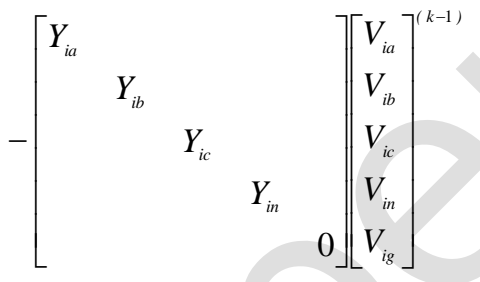

where

$I_{i a}, I_{i b}, I_{i c}, I_{i n}, I_{i g}$ $S_{i a}, S_{i b}, S_{i c}$

$V_{i a}, V_{i b}, V_{i c}, V_{i n}, V_{i g}$

$Y_{i a}, Y_{i b}, Y_{i c}, Y_{i n}$

$Z_{g r i}$

are current injections at node $i$; are scheduled (known) power injections at node $i$; are voltages at node $i$; are admittances of all shunt elements at node $i$; and is grounding impedance at node $i\left(Z_{g i}=\right.$ $Z_{g r i}+Z_{g g i}$.

2. Backward sweep - section current calculation

Starting from the line section in the last layer and moving towards the root node, the current in line section $l$ is:

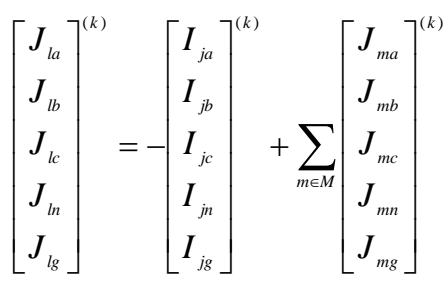

where

$J_{l a}, J_{l b}, J_{l c}, J_{l n}, J_{l g}$ are the current flows on line section $l$; and $M \quad$ is the set of line sections connected downstream to node $j$.

3. Forward sweep - nodal voltage calculation

Starting from the first layer and moving towards the last layer, the voltage at node $j$ is:

$$
\left[\begin{array}{l}
V_{j a} \\
V_{j b} \\
V_{j c} \\
V_{j n} \\
V_{j g}
\end{array}\right]^{(k)}=\left[\begin{array}{c}
V_{i a} \\
V_{i b}^{(k)} \\
V_{i c} \\
V_{i n} \\
V_{i g}
\end{array}\right]^{(k)}-\left[\begin{array}{lllll}
Z_{a a} & Z_{a b} & Z_{a c} & Z_{a n} & Z_{a g} \\
Z_{a b} & Z_{b b} & Z_{b c} & Z_{b n} & Z_{b g} \\
Z_{a c} & Z_{b c} & Z_{c c} & Z_{c n} & Z_{c g} \\
Z_{a n} & Z_{b n} & Z_{c n} & Z_{n n} & Z_{n g} \\
Z_{a g} & Z_{b g} & Z_{c g} & Z_{n g} & Z_{g g}
\end{array}\right]\left[\begin{array}{l}
J_{l a} \\
J_{l b} \\
J_{l c} \\
J_{l n} \\
J_{l g}
\end{array}\right]^{(k)}
$$

\section{Voltage correction}

Voltage correction in the nodes with grounded neutral and downstream nodes should be performed. Voltage in the nodes with neutral grounded at iteration $k$ is as follows:

$$
V_{i n}^{(k)}=Z_{g r i} J_{l g}^{(k)}, i \in\{\text { Grounded nodes }\}
$$

Convergence criterion

After these steps are executed in one iteration, the power 
mismatches at each node for all phases, neutral wire and ground are calculated:

$$
\begin{aligned}
& \Delta S_{i a}^{(k)}=V_{i a}^{(k)}\left(I_{i a}^{(k)}\right)^{*}-Y_{i a}^{*}\left|V_{i a}^{(k)}\right|^{2}-S_{i a} \\
& \Delta S_{i b}^{(k)}=V_{i b}^{(k)}\left(I_{i b}^{(k)}\right)^{*}-Y_{i b}^{*}\left|V_{i b}^{(k)}\right|^{2}-S_{i b} \\
& \Delta S_{i c}^{(k)}=V_{i c}^{(k)}\left(I_{i c}^{(k)}\right)^{*}-Y_{i c}^{*}\left|V_{i c}^{(k)}\right|^{2}-S_{i c} \\
& \Delta S_{i n}^{(k)}=V_{i n}^{(k)}\left(I_{i n}^{(k)}\right)^{*}-Y_{i n}^{*}\left|V_{i n}^{(k)}\right|^{2} \\
& \Delta S_{i g}^{(k)}=V_{i g}^{(k)}\left(I_{i g}^{(k)}\right)^{*}
\end{aligned}
$$

If the real or imaginary part of any of the power mismatches is greater than a convergence criterion, steps 1,2 and 3 are repeated until convergence is achieved.

Flat start

The initial voltage for all nodes should be equal to the root node voltage:

$$
\left[\begin{array}{c}
V_{i a} \\
V_{i b} \\
V_{i c} \\
V_{i n} \\
V_{i g}
\end{array}\right]^{(0)}=\left[\begin{array}{c}
V_{r e f} \\
a^{2} \cdot V_{r e f} \\
a \cdot V_{r e f} \\
0 \\
0
\end{array}\right], \quad a=e^{j \frac{2 \pi}{3}} .
$$

Equations for calculation of the impedance matrix in a $5 \times 5$ representation are given in the Appendix. Most of the existing models of three-phase elements of the distribution network (lines, loads, shunt capacitors, distribution transformers, dispersed generators) can be applied in the proposed $5 \times 5$ power flow method according to the general form given in [15].

\section{TEST NETWORKS}

The proposed power flow method using a $5 \times 5$ network representation is applied on numerous MV and LV DNs. In this paper the following networks are used:

A. Four-wire three-phase MV feeder with isolated neutral (without ground conductor),

B. Three-phase MV line with earth return (without neutral wire), and

C. Four-wire three-phase LV distribution network.

The test network used in cases A and B is the IEEE 34-bus radial distribution network [17] and [18], Fig. 3. Base voltage of the network is $V_{b}=24.9 \mathrm{kV}$, and the reference voltage in the root node is $V_{\text {ref }}=25.647 \mathrm{kV}$. Simplifying, the autotransformer $24.9 / 4.16 \mathrm{kV} / \mathrm{kV}$ in the original IEEE 34-bus test feeder is replaced with the line and the network is modeled with the single voltage level. The automatic voltage regulator is also not represented. Mutual and self ground impedances $Z_{a g}, Z_{b g}, Z_{\mathrm{cg}}, Z_{\mathrm{ng}}$ and $Z_{\mathrm{gg}}$ (elements of the fifth row and fifth column of the $5 \times 5$ matrix) are computed as shown in Appendix. The values for the IEEE 34-bus DN are shown in Table I. It should be noted that mutual and self impedances of phases $a, b, c$ and neutral (additional elements of the 5x5) are performed using Carson's equations, considering the ground as a perfect conductor [2]. For space reason, it is solely presented in Table II the 5x5 matrix for the 1-2 line section. The assumed ground conductor impedance is developed based on voltage drop equations for the three-phase line with earth return [2] and full Carson's impedance calculation [16].

\begin{tabular}{|c|c|c|c|c|c|c|}
\hline SD & $\mathrm{RV}$ & $\mathrm{Z}_{\mathrm{ag}}(\Omega)$ & $\mathrm{Z}_{\mathrm{bg}}(\Omega)$ & $\mathrm{Z}_{\mathrm{cg}}(\Omega)$ & $\mathrm{Z}_{\mathrm{ng}}(\Omega)$ & $Z_{g g}(\Omega)$ \\
\hline 0 & 1 & j 0.0560 & 0.0560 & j 0.0560 & j 0.0514 & $0.0466+\mathrm{j} 0.3437$ \\
\hline 1 & 2 & j 0.0375 & j 0.0375 & j 0.0375 & j 0.0345 & $0.0312+j 0.2305$ \\
\hline 2 & 3 & j 0.6994 & j 0.6994 & j 0.6994 & 0.6425 & $0.5816+j 4.2939$ \\
\hline 3 & 4 & j 0.0000 & 0.1283 & j 0.0000 & 0.1157 & $0.1047+j 0.7733$ \\
\hline 3 & 5 & j 0.8138 & 0.8138 & 0.8138 & 0.7475 & $0.6767+j 4.9961$ \\
\hline 5 & 6 & j 0.6452 & 0.6452 & 0.6452 & 0.5926 & $0.5365+j 3.9609$ \\
\hline 6 & 7 & j 0.0002 & 0.0002 & j 0.0002 & 0.0002 & $0.0002+\mathrm{j} 0.0013$ \\
\hline 7 & 8 & j 0.0067 & 0.0067 & j 0.0067 & 0.0062 & $0.0056+j 0.0413$ \\
\hline 8 & 9 & j 0.0378 & 0.0000 & j 0.0000 & 0.0341 & $0.0309+j 0.2278$ \\
\hline 8 & 10 & j 0.2216 & 0.2216 & 0.2216 & 0.2035 & $0.1842+j 1.3603$ \\
\hline 9 & \begin{tabular}{|l|}
11 \\
\end{tabular} & j 1.0640 & 0.0000 & j 0.0000 & 0.9598 & $0.8688+j 6.4149$ \\
\hline 10 & 12 & j 0.0182 & 0.0182 & j 0.0182 & 0.0167 & $0.0152+\mathrm{j} 0.1119$ \\
\hline 10 & \begin{tabular}{|l|}
13 \\
\end{tabular} & j 0.0000 & 0.0670 & j 0.0000 & 0.0604 & $0.0547+\mathrm{j} 0.4037$ \\
\hline 11 & 14 & j 0.3036 & 0.0000 & j 0.0000 & 0.2739 & $0.2479+j 1.8306$ \\
\hline 12 & 15 & j 0.4436 & 0.4436 & j 0.4436 & 0.4074 & $0.3688+j 2.7232$ \\
\hline 15 & \begin{tabular}{|l|}
16 \\
\end{tabular} & j 0.0113 & 0.0113 & j 0.0113 & 0.0104 & $0.0094+j 0.0693$ \\
\hline 16 & 17 & j 0.7993 & 0.7993 & j 0.7993 & 0.7342 & $0.6646+j 4.9068$ \\
\hline 16 & \begin{tabular}{|l|}
18 \\
\end{tabular} & j 0.0000 & 0.5155 & j 0.0000 & 0.4651 & $0.4210+j 3.1082$ \\
\hline 17 & \begin{tabular}{|l|}
19 \\
\end{tabular} & j 0.0002 & 0.0002 & j 0.0002 & 0.0002 & $0.0002+j 0.0013$ \\
\hline 19 & 20 & j 0.0000 & 0.0000 & j 0.0000 & 0.0000 & $0.0000+j 0.0000$ \\
\hline 19 & 21 & j 0.1063 & 0.1063 & j 0.1063 & j 0.0977 & $0.0884+j 0.6528$ \\
\hline 20 & 22 & j 0.2292 & 0.2292 & j 0.2292 & 0.2105 & $0.1905+j 1.4069$ \\
\hline 21 & \begin{tabular}{|l|}
23 \\
\end{tabular} & j 0.1265 & 0.1265 & j 0.1265 & 0.1162 & $0.1052+\mathrm{j} 0.7767$ \\
\hline 21 & 24 & j 0.0000 & 0.0358 & j 0.0000 & 0.0323 & $0.0292+j 0.2158$ \\
\hline 23 & 25 & j 0.0438 & 0.0438 & j 0.0438 & 0.0403 & $0.0364+j 0.2691$ \\
\hline 23 & 26 & j 0.0061 & 0.0061 & j 0.0061 & 0.0056 & $0.0051+\mathrm{j} 0.0373$ \\
\hline 25 & 27 & j 0.0582 & 0.0582 & 0.0582 & 0.0534 & $0.0484+j 0.3571$ \\
\hline 26 & 28 & j 0.0293 & 0.0293 & 0.0293 & 0.0269 & $0.0244+j 0.1799$ \\
\hline 27 & 29 & j 0.0061 & 0.0061 & 0.0061 & 0.0056 & $0.0051+j 0.0373$ \\
\hline 27 & 30 & j 0.0187 & 0.0187 & j 0.0187 & 0.0171 & $0.0155+\mathrm{j} 0.1146$ \\
\hline 28 & 31 & j 0.0790 & 0.0790 & j 0.0790 & 0.0726 & $0.0657+\mathrm{j} 0.4850$ \\
\hline 29 & 32 & j 0.0000 & 0.1074 & j 0.0000 & j 0.0969 & $0.0877+\mathrm{j} 0.6475$ \\
\hline 31 & 33 & j 0.0115 & 0.0115 & j 0.0115 & j 0.0106 & $0.0096+j 0.0706$ \\
\hline
\end{tabular}

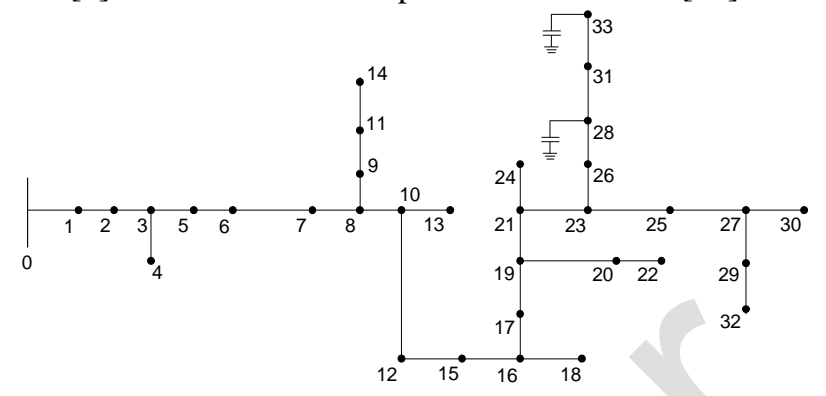

Fig. 3. IEEE 34-bus MV test feeder, Cases A and B.

TABLE I

MUTUAL AND SELF GROUND IMPEDANCE - IEEE 34-BUS DN (SD: SENDING BUS AND RV: RECEIVING BUS)

TABLE II

5 X5 IMPEDANCE MATRIX FOR THE 1-2 LINE SECTION $(\Omega)$

\begin{tabular}{|c|c|c|c|c|}
\hline $0.5537+\mathrm{j} 0.3778$ & $\mathrm{j} 0.1237$ & $\mathrm{j} 0.1003$ & $\mathrm{j} 0.0994$ & $\mathrm{j} 0.0375$ \\
\hline $\mathrm{j} 0.1237$ & $0.5537+\mathrm{j} 0.3778$ & $\mathrm{j} 0.0830$ & $\mathrm{j} 0.0883$ & $\mathrm{j} 0.0375$ \\
\hline $\mathrm{j} 0.1003$ & $\mathrm{j} 0.0830$ & $0.5537+\mathrm{j} 0.3778$ & $\mathrm{j} 0.0932$ & $\mathrm{j} 0.0375$ \\
\hline $\mathrm{j} 0.0994$ & $\mathrm{j} 0.0883$ & $\mathrm{j} 0.0932$ & $0.5537+\mathrm{j} 0.3717$ & $\mathrm{j} 0.0345$ \\
\hline $\mathrm{j} 0.0375$ & $\mathrm{j} 0.0375$ & $\mathrm{j} 0.0375$ & $\mathrm{j} 0.0345$ & $0.0312+\mathrm{j} 0.2305$ \\
\hline
\end{tabular}

Case A considers an isolated neutral; therefore the fifth row and fifth column contain all zero elements. Since in case B the neutral wire does not exist, the fourth row and fourth column of the 5x5 impedance matrix contain all zero entries. All impedances calculations use a $100 \Omega$-m ground resistivity (except where ground resistivity is a parameter).

Test network used in case $\mathrm{C}$ is the 29-node radial real-life distribution network (LV-29 DN), Fig. 4. The base voltage of the network is $V_{b}=121.244 \mathrm{~V}$. Real and reactive load injections are shown in Table III.

Four cases of LV network are analyzed:

C1. LV network with isolated neutral (without ground conductor);

C2. Multi-grounded LV network, grounding resistance $R_{g r}$ $=0.2 \Omega$ in the following nodes: $3,4,11,16,17,18,25$, 
$28, R_{g r}=0.1 \Omega$ in substation MV/LV;

C3. Same as in case $\mathrm{C} 2$, but $R_{g r}=0.5 \Omega$;

C4. LV network with all nodes grounded, $R_{g r}=0.1 \Omega$.

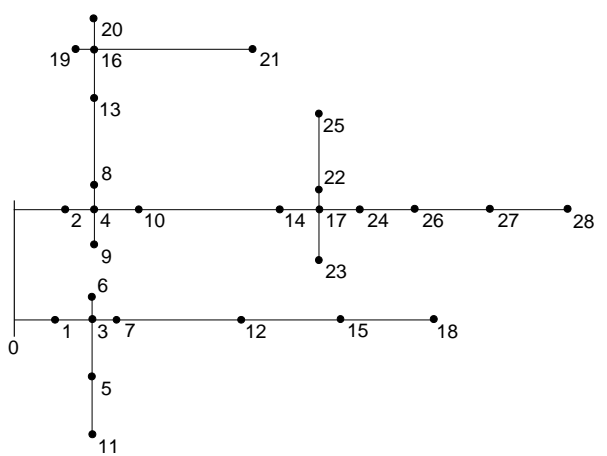

Fig. 4. LV-29 test feeder, Case C.

TABLE III

REAL AND REACTIVE LOAD INJECTIONS - LV-29 DN (NON-ZERO ELEMENTS)

\begin{tabular}{|c|r|r|r|r|r|r|}
\hline node & $\mathrm{P}_{\mathrm{La}}(\mathrm{W})$ & \multicolumn{1}{|c|}{$\mathrm{Q}_{\mathrm{La}}(\mathrm{VAr})$} & \multicolumn{1}{|c|}{$\mathrm{P}_{\mathrm{Lb}}(\mathrm{W})$} & $\mathrm{Q}_{\mathrm{Lb}}(\mathrm{VAr})$ & $\mathrm{P}_{\mathrm{Lc}}(\mathrm{W})$ & $\mathrm{Q}_{\mathrm{Lc}}(\mathrm{VAr})$ \\
\hline 2 & 451.4 & 148.4 & 209.7 & 68.9 & 0.0 & 0.0 \\
\hline 5 & 0.0 & 0.0 & 18.1 & 5.9 & 329.2 & 108.2 \\
\hline 7 & 641.7 & 210.9 & 397.9 & 130.8 & 504.9 & 166.0 \\
\hline 8 & 188.2 & 61.9 & 268.8 & 88.4 & 0.0 & 0.0 \\
\hline 9 & 166.0 & 54.6 & 97.2 & 31.9 & 349.3 & 114.8 \\
\hline 11 & 262.5 & 86.3 & 142.4 & 46.8 & 120.1 & 39.5 \\
\hline 12 & 326.4 & 107.3 & 441.0 & 144.9 & 331.3 & 108.9 \\
\hline 13 & 147.2 & 48.4 & 310.4 & 102.0 & 127.1 & 41.8 \\
\hline 14 & 377.1 & 123.9 & 348.6 & 114.6 & 403.5 & 132.6 \\
\hline 15 & 1044.4 & 343.3 & 1185.4 & 389.6 & 1020.1 & 335.3 \\
\hline 18 & 330.6 & 108.7 & 562.5 & 184.9 & 754.2 & 247.9 \\
\hline 19 & 0.0 & 0.0 & 236.8 & 77.8 & 216.0 & 71.0 \\
\hline 20 & 311.1 & 102.3 & 544.4 & 178.9 & 234.7 & 77.1 \\
\hline 21 & 294.4 & 96.8 & 59.7 & 19.6 & 0.0 & 0.0 \\
\hline 22 & 112.5 & 37.0 & 112.5 & 37.0 & 0.0 & 0.0 \\
\hline 23 & 113.9 & 37.4 & 0.0 & 0.0 & 0.0 & 0.0 \\
\hline 24 & 0.0 & 0.0 & 170.8 & 56.1 & 170.8 & 56.1 \\
\hline 25 & 570.8 & 187.6 & 273.6 & 89.9 & 0.0 & 0.0 \\
\hline 26 & 507.6 & 166.8 & 291.0 & 95.6 & 343.1 & 112.8 \\
\hline 27 & 144.4 & 47.5 & 366.7 & 120.5 & 569.4 & 187.2 \\
\hline 28 & 118.1 & 38.8 & 118.1 & 38.8 & 0.0 & 0.0 \\
\hline
\end{tabular}

\section{APPLICATIONS}

The proposed method for distribution power flow is tested on numerous MV and LV real life DNs. In this paper, the proposed power flow in a $5 \times 5$ network representation is applied on three DNs. Two types of load models are applied: constant admittance and constant power.

Results obtained by performing the proposed power flow method on IEEE 34-bus DN, case A, are shown from Fig. 5 to Fig. 9. Figure 5 shows voltages of the neutral wire in the fourwire three-phase IEEE 34-bus network when the loads are modeled as a constant power or constant admittance. The neutral voltage at the end node $(275 \mathrm{~V})$ is generated by the flow of the return current in the neutral wire, Fig.5. Figures 6 and 7 show phase voltages when the loads are modeled as a constant admittance or constant power, respectively. Figure 8 shows voltage unbalance in case $\mathrm{A}$, when the loads are modeled as a constant power or constant admittance load, according to the IEEE Std. 446. Figure 9 shows neutral current in the main path in case A, for constant power and constant admittance load models. Since in the proposed model neutral wire and ground are explicitly represented, it is possible to calculate power losses in the neutral wire and in the ground. Real power losses in the neutral wire in the considered IEEE 34-bus DN are about 3\% of the total real power losses.
Results from the use of the suggested power flow method on IEEE 34-bus DN with earth return, case B, are shown on Fig. 10 to Fig. 16. Differences in phase voltages obtained from the three-phase power flow method [10] and the proposed method ranged $1.5 \%$ (Fig. 10, Fig. 13). Figures 14 and 15 show ground voltages after modeling for different ground resistivity $(50-5000 \Omega-\mathrm{m})$, for constant power and constant admittance load models, respectively [2]. Figure 16 shows ground current in the three-phase MV line with earth return.

The results obtained by performing the proposed power flow method on LV-29 DN are shown on Fig. 17 to Fig. 24. The differences in phase voltages obtained from the conventional three-phase power flow [10] and the proposed method were up to $0.2 \%$ (Fig. 17, Fig. 18). The neutral voltage at the end node is induced by the flow of the return current in the neutral conductor (case $\mathrm{C} 1$ ) and by the ground impedance in the case of a multi-grounded LV network (Fig. 20 ). When it is difficult to make a proper grounding, most of the current flows through the neutral conductor. A high neutral voltage at the end node may cause not only common-mode noise problems in sensitive electronic pieces of equipment but it may also represent a safety issue [4]. The voltage unbalance, according to the IEEE Std. 446, is less than $0.3 \%$ in all cases (Fig. 21). Figures 23 and 24 show respectively neutral wire and ground currents in the evaluated LV-29 DN, when the loads are modeled as constant admittance. The current in the neutral wire is about 30\% of the phase currents in the considered LV-29 DN.

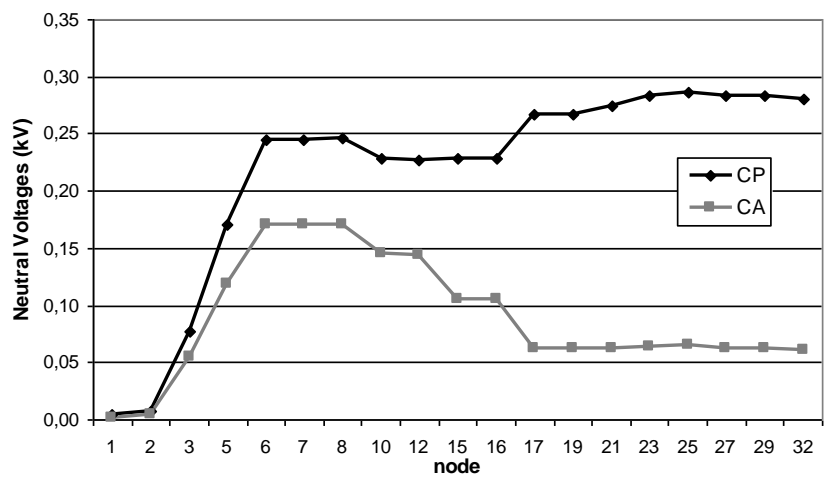

Fig. 5. Neutral Voltage: Case A with Constant Power (CP) and Constant Admittance (CA) Load Model.

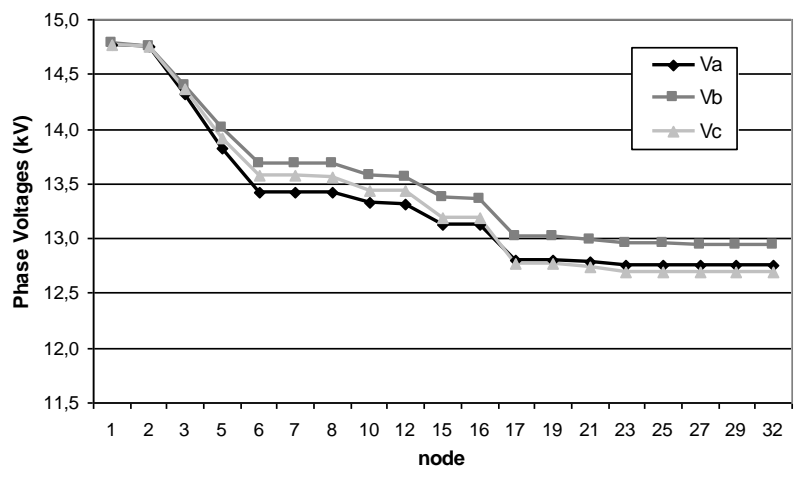

Fig. 6. Phase Voltages: Case A with Constant Admittance Load Model. 


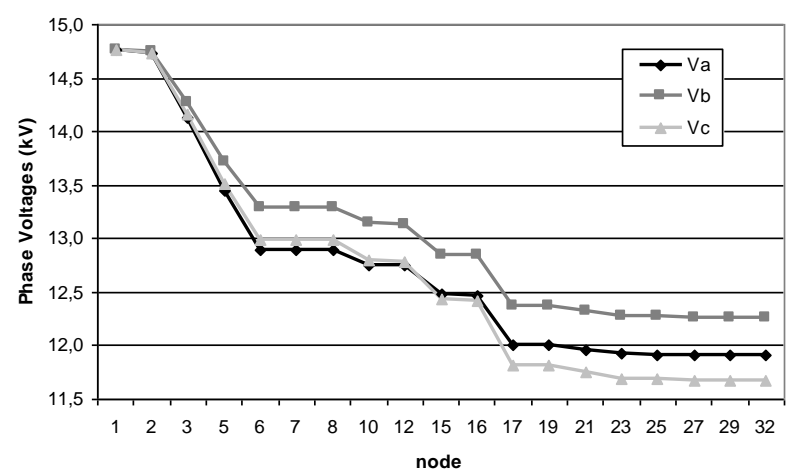

Fig. 7. Phase Voltages: Case A with Constant Power Load Model.

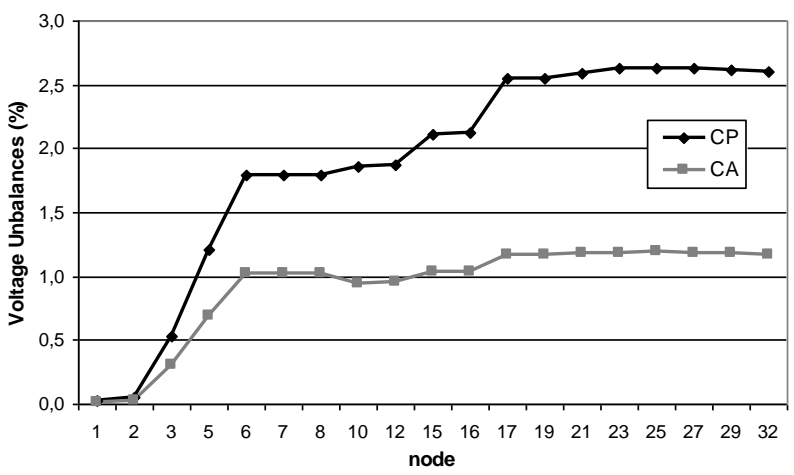

Fig. 8. Voltage Unbalance: Case A with Constant Power (CP) and Constant Admittance (CA) Load Model according to the IEEE Std. 446.

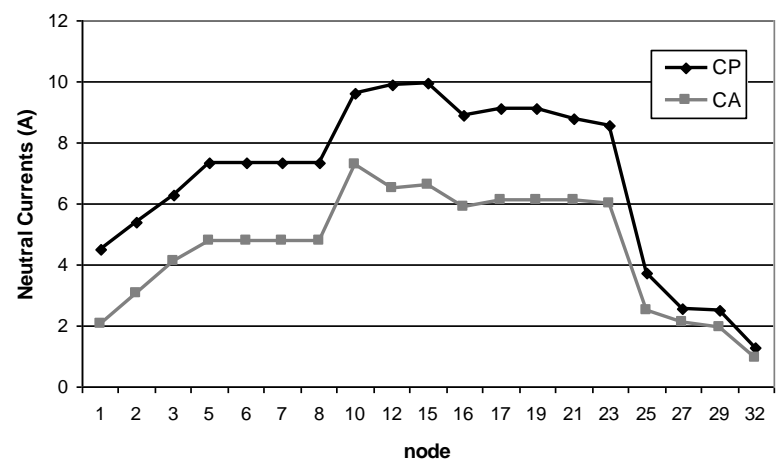

Fig. 9. Neutral current: Case A with Constant Power (CP) and Constant Admittance (CA) Load Model.

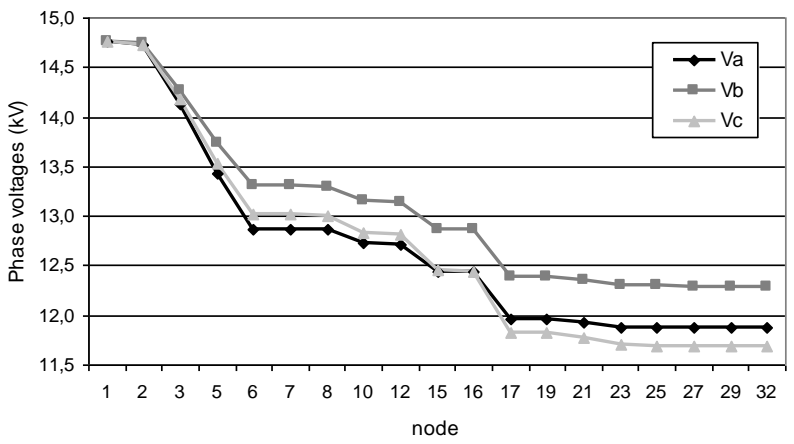

Fig. 10. Phase Voltages using proposed power flow method: Case B with Constant Power Load Model.

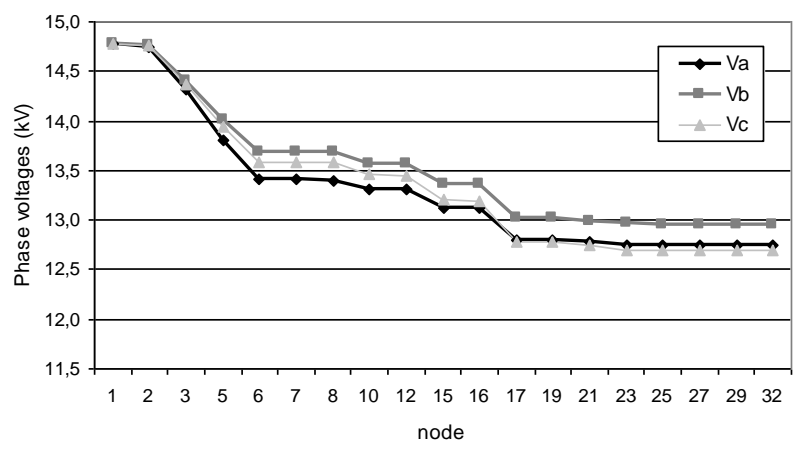

Fig. 11. Phase Voltages using proposed power flow method: Case B with Constant Admittance Load Model.

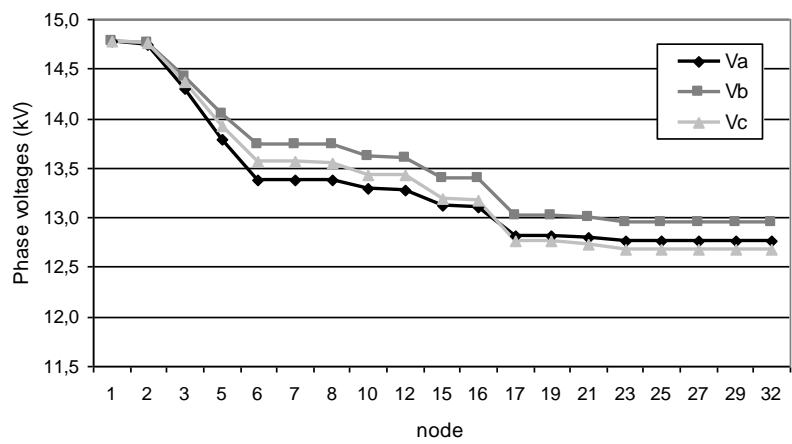

Fig. 12. Phase Voltages using ordinary three-phase power flow method: Case B with Constant Admittance Load Model.

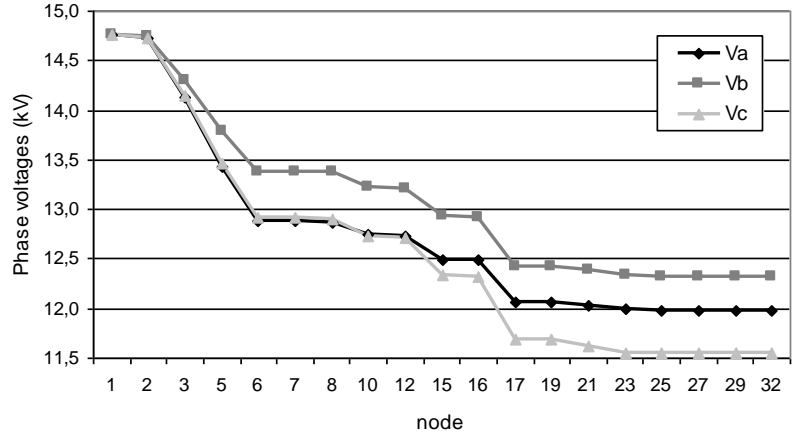

Fig. 13. Phase Voltages using ordinary three-phase power flow method: Case B with Constant Power Load Model.

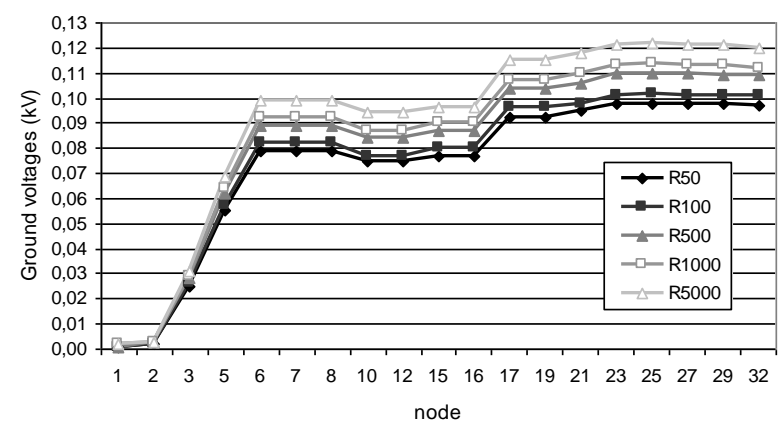

Fig. 14. Ground Voltages: Case B with different ground resistance and Constant Power Load Model. 


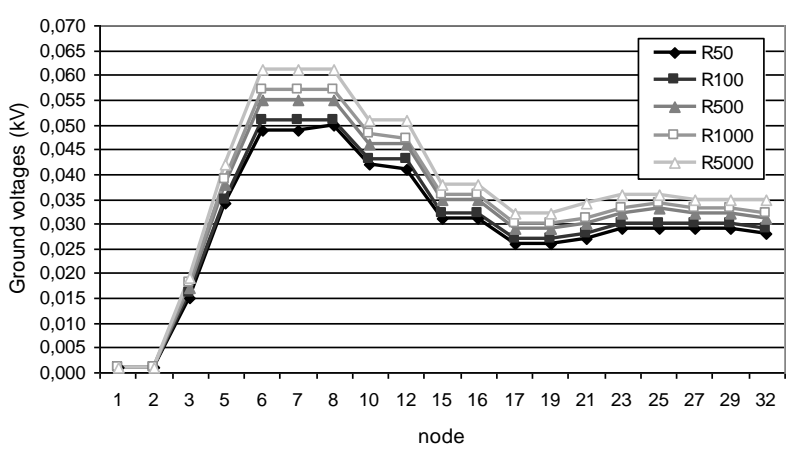

Fig. 15. Ground Voltages: Case B with different ground resistance and Constant Admittance Load Model.

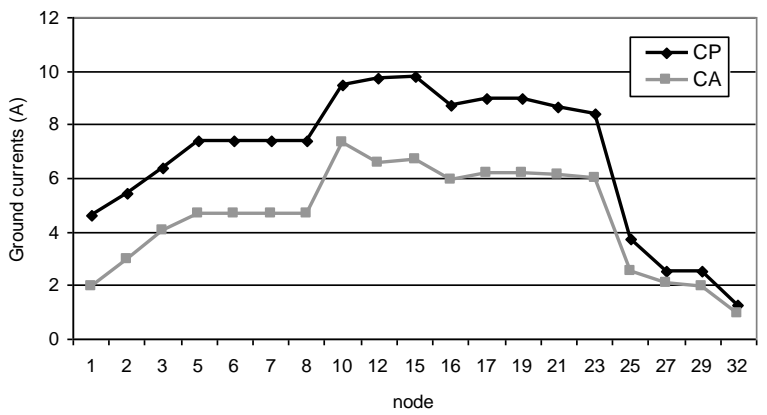

Fig. 16. Ground Current: Case B with Constant Power (CP) and Constant Admittance (CA) Load Model.

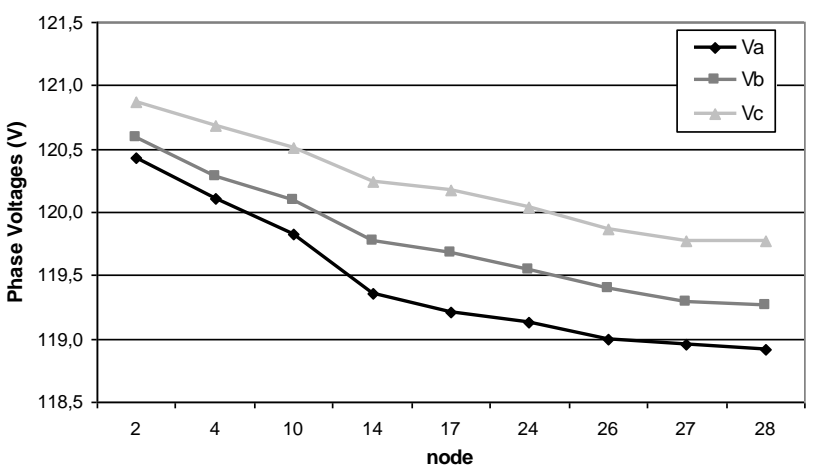

Fig. 17. Phase Voltages using conventional three-phase power flow method: Case C with Constant Admittance Load Model.

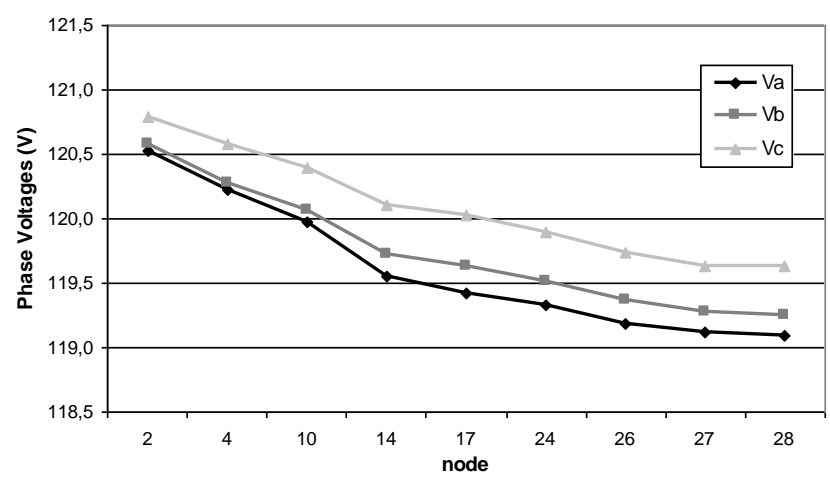

Fig. 18. Phase Voltages using proposed power flow method: Case C with Constant Admittance Load Model and Isolated Neutral (C1).

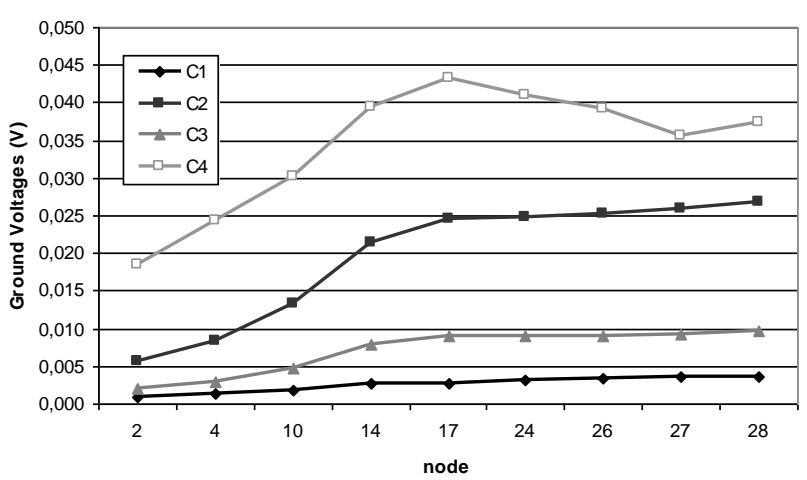

Fig. 19. Ground Voltages: Case C with Constant Admittance Load Model.

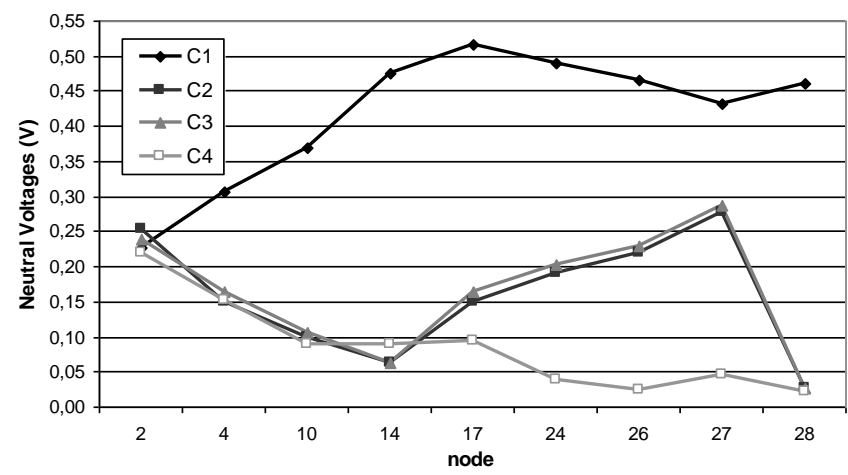

Fig. 20. Neutral Voltages: Case C with Constant Admittance Load Model.

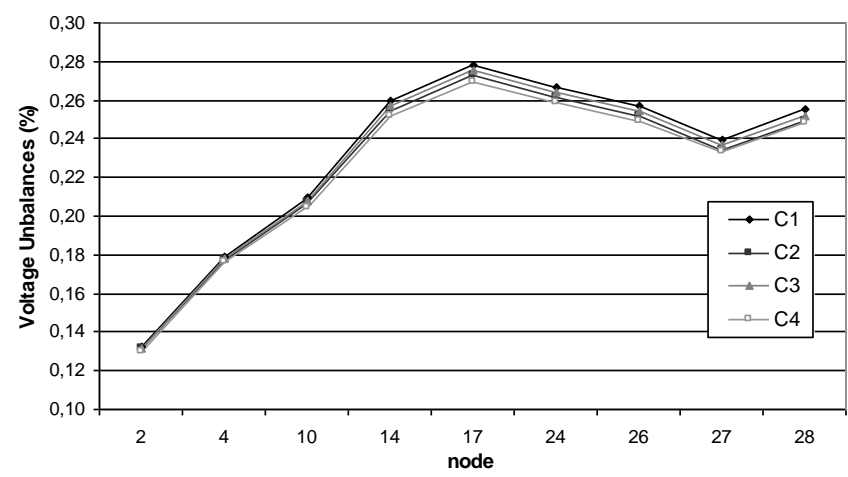

Fig. 21. Voltage Unbalances: Case C with Constant Admittance Load Model according to the IEEE Std. 446.

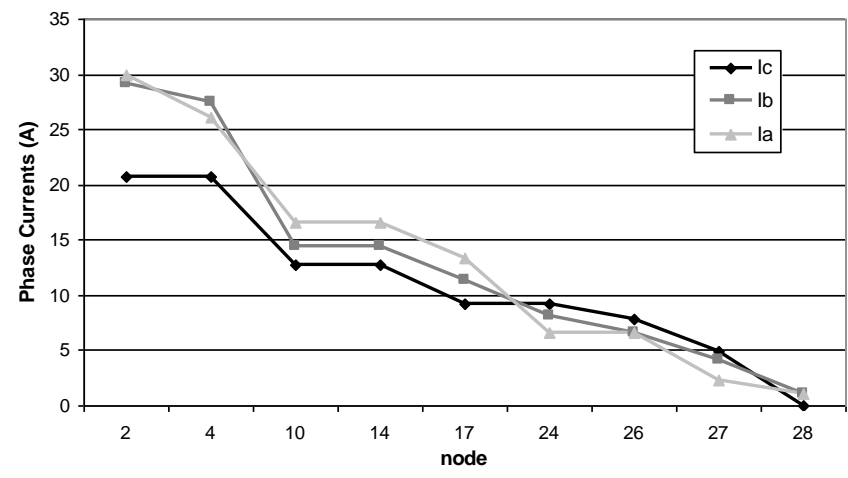

Fig. 22. Phase Currents: Case $\mathrm{C}$ with Constant Admittance Load Model and Isolated Neutral (C1). 


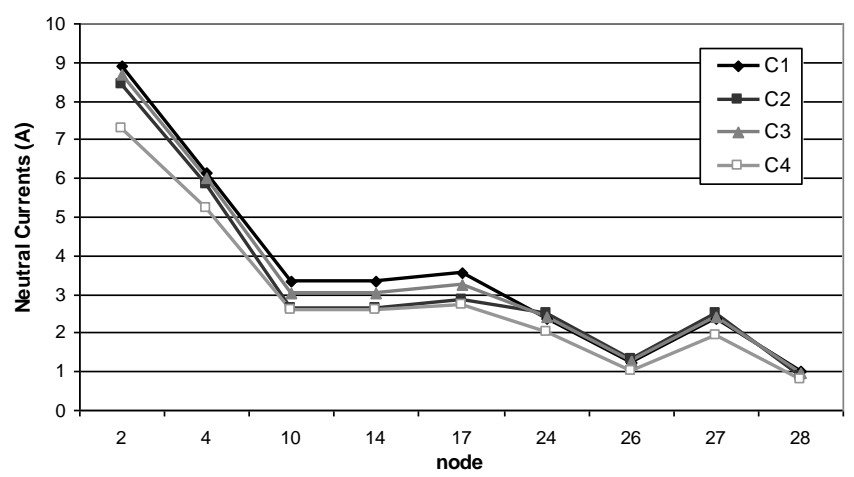

Fig. 23. Return currents: Case C with Constant Admittance Load Model.

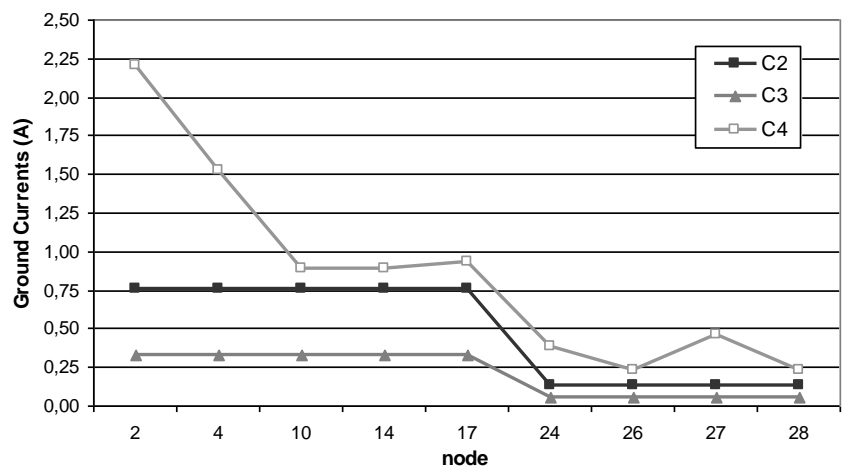

Fig. 24. Ground Currents: Case C with Constant Admittance Load Model.

Generally, load model plays a significant role in all power flow simulations. The results showed a large difference in the phase voltages due to the load model $(7.0 \%)$, and a minor difference as a function of the line model $(1.5 \%)$. The offdiagonal shunt admittances were neglected in all simulations, as stated in (1). However, the influence of the mutual shunt admittances on the solution was also investigated. Considering Case A, when the neutral wire and ground were explicitly represented, the difference in phase voltages with and without off-diagonal shunt admittance terms in (1), was up to $0.95 \%$.

Results showed that Kron's reduction is acceptable when neutral and ground current are not of specific interest. Moreover, the return currents may be calculated using $3 \times 3$ approach, after which the voltages in return paths may be obtained using the original $5 \times 5$ matrices. Kron's reduction is avoided with this proposed method when the focus is to calculate currents and voltages in an explicit manner.

The proposed method was tested on MV real-life network 475 nodes in Assis (Sao Paulo state, Brazil). When the peak load case was simulated, the $5 \times 5$ and the $3 \times 3$ backwardforward techniques converged after three iterations, with a computing time of 770 and 430 milliseconds, respectively, when a $544 \mathrm{MHz}$ computer processor was used.

\section{CONCLUSIONS}

In this paper, a power flow algorithm for three-phase fourwire radial DNs, considering neutral wire and multigrounding, is proposed. High-order line models $(4 \times 4,5 \times 5$ and higher) may easily be added to a common solution method for three-phase power flow [10]. Most of the existing models of three-phase elements of the distribution system can be well represented [15]. The introduced $5 \times 5$ network representation can easily be incorporated into a power flow solution technique for weakly meshed DNs with PV nodes. The proposed power flow method enables the user to investigate the effects of neutrals and system grounding on the operation of real-life DNs.

The software package based on the proposed $5 \times 5$ power flow solution technique can be useful for the evaluation of different neutral designs. Moreover, it can be an efficient tool for power quality studies, safety analysis, short circuit analysis, or other applications, where neutral and ground currents and voltages are of particular interest [19], [20].

\section{APPENDIX}

The equations for calculation of impedance matrix elements in $5 \times 5$ representation are as follows.

Using the ground return modeling of [2] for a three-phase line (Fig. A), we obtain:

$$
\left[\begin{array}{l}
V_{a} \\
V_{b} \\
V_{c}
\end{array}\right]=\left[\begin{array}{lll}
z_{a a} & z_{a b} & z_{a c} \\
z_{a b} & z_{b b} & z_{b c} \\
z_{a c} & z_{b c} & z_{c c}
\end{array}\right]\left[\begin{array}{l}
I_{a} \\
I_{b} \\
I_{c}
\end{array}\right]
$$

where

$$
\begin{aligned}
& z_{x x}=\bar{z}_{x x}+\bar{z}_{g g}-2 \bar{z}_{x g} \\
& z_{x y}=\bar{z}_{x y}-\bar{z}_{x g}-\bar{z}_{y g}+\bar{z}_{g g} \\
& x=a, b, c ; y=a, b, c ; \forall \quad \forall \neq \mathrm{y}
\end{aligned}
$$

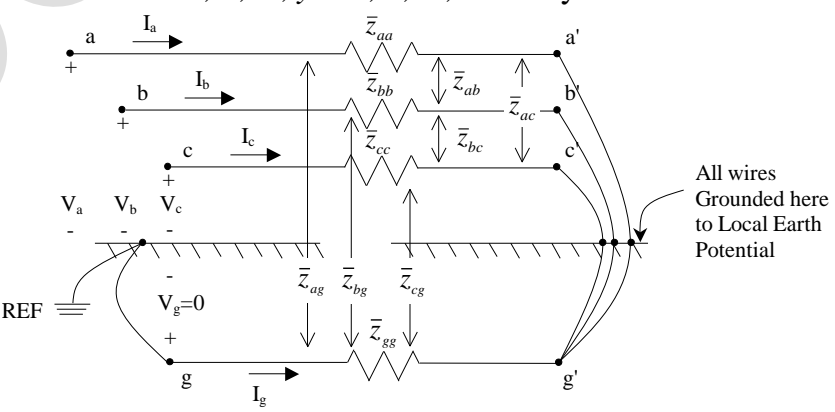

Fig. A. Three-phase line with earth return [2].

In the case of wire $a, \bar{z}_{a a}$ represents the self-impedance of the line (if the ground is a perfect conductor), and the term $\left(\bar{z}_{g g}-2 \bar{z}_{a g}\right)$ is the correction caused by the ground influence.

Using the effect of the finite conductivity of the ground (correction part) of the simplified Carson's equations for selfimpedance, we obtain the following equation:

$$
\begin{aligned}
& \bar{z}_{g g}-2 \bar{z}_{a g}=\pi^{2} \cdot 10^{-4} f-j 0.0386 \cdot 8 \pi \cdot 10^{-4} f \\
& +j 4 \pi \cdot 10^{-4} \cdot f \ln \frac{2}{5.6198 \cdot 10^{-3}}+j 4 \pi \cdot 10^{-4} \cdot f \ln \frac{\sqrt{\rho / f}}{h_{a}} \Omega / \mathrm{km}(i v)
\end{aligned}
$$

where

$f \quad$ is the frequency $(\mathrm{Hz})$;

$h_{a} \quad$ is the height of wire (m);

$\rho \quad$ is the ground resistivity $(\Omega-m)$.

Looking at the right side of (iv), we can easily identify the 
terms that are only frequency dependent. These three terms represent the ground self-impedance $\bar{z}_{g g}$. Therefore, the ground self-impedance $\bar{z}_{g g}$ and the mutual impedance $\bar{z}_{a g}$ from Fig. A and (iv) are, respectively

$$
\begin{aligned}
\bar{z}_{g g} & =\pi^{2} \cdot 10^{-4} f-j 0.0386 \cdot 8 \pi \cdot 10^{-4} f \\
+j 4 \pi \cdot 10^{-4} \cdot f \ln \frac{2}{5.6198 \cdot 10^{-3}} & \Omega / \mathrm{km} \\
\bar{z}_{a g} & =-\frac{1}{2} \cdot j 4 \pi \cdot 10^{-4} \cdot f \ln \frac{\sqrt{\rho / f}}{h_{a}} \Omega / \mathrm{km} \\
& =j 2 \pi \cdot 10^{-4} \cdot f \ln \frac{h_{a}}{\sqrt{\rho / f}}
\end{aligned}
$$

Hence, for a three-phase line with earth return, the $5 \times 5$ matrix representation of the line is as follows:

$$
\left[Z_{\text {line }}\right]=\left[\begin{array}{ccccc}
\bar{z}_{a a} & \bar{z}_{a b} & \bar{z}_{a c} & 0 & \bar{z}_{a g} \\
\bar{z}_{a b} & \bar{z}_{b b} & \bar{z}_{b c} & 0 & \bar{z}_{b g} \\
\bar{z}_{a c} & \bar{z}_{b c} & \bar{z}_{c c} & 0 & \bar{z}_{c g} \\
0 & 0 & 0 & 0 & 0 \\
\bar{z}_{a g} & \bar{z}_{b g} & \bar{z}_{c g} & 0 & \bar{z}_{g g}
\end{array}\right]
$$

The self and mutual impedances of phases $a, b$ and $c$ in (vii), are calculated using the Carson's equations, considering the ground as a perfect conductor [2]. Self and mutual impedances of the ground use $(v)$ and $(v i)$. Zero elements on matrix (vii) are due to the absence of neutral wire.

For neutral wire calculations, mutual ground impedance should be computed using (vi) and mutual $a, b, c$ and self impedances should be computed using the Carson's equations, considering the ground as a perfect conductor [2].

\section{ACKNOWLEDGMENT}

The authors would like to thank UNESP - Ilha Solteira, SP, Brazil, for the use of their facilities. R. M. Ciric is grateful to the Fundação de Amparo à Pesquisa do Estado de São Paulo (FAPESP) for its financial support to undertake this research (Project no. 00/12099-1).

\section{REFERENCES}

[1] E. Lakervi and E. J. Holmes, Electricity Distribution Network Design, IEE Power Engineering Series 21, London: Peter Peregrines Ltd. On behalf of the IEE, 1995.

[2] P. M. Anderson, Analysis of Faulted Power Systems, New York: IEEE Press Power Systems Engineering Series, 1995, pp. 71-83.

[3] A. P. Meliopoulos, J. Kennedy, C. A. Nucci, A. Borghetti, and G. Contaxies, "Power distribution practices in USA and Europe: Impact on power quality," in Proc. 1998 International Conference on Harmonics and Quality of Power, vol. 1, pp. 24-29.

[4] T. H. Chen, and W. C. Yang, "Analysis of multi-grounded four-wire distribution systems considering the neutral grounding," IEEE Trans. Power Delivery, vol. 16, no. 4, pp. 710-717, Oct. 2001.

[5] T. A. Short, J. R. Stewart, D. R. Smith, J. O’Brien, and K. Hampton, "Five-Wire Distribution System Demonstration Project, " IEEE Trans. Power Delivery, vol. 17, no. 2, pp. 649-654, Apr. 2002.

[6] J. C. Balda, A. R. Oliva, D. W. McNabb, and R. D. Richardson, "Measurements of neutral currents and voltages on a distribution feeder," IEEE Trans. Power Delivery, vol. 12, no. 4, pp. 1799-1804, Oct. 1997.
[7] J. C. Das and R. H. Osman, "Grounding of AC and DC low-voltage and medium-voltage drive system," IEEE Trans. Industry Applications, vol. 34, no. 1, pp. 205-216, Jan./Feb. 1998.

[8] IEEE Recommended Practice for Grounding of Industrial and Commercial Power Systems, IEEE Standard 142-1991, Jun. 1992.

[9] D. Shirmohammadi, H. W. Hong, A. Semlyen, and G. X. Luo, "A compensation-based power flow method for weakly meshed distribution and transmission networks," IEEE Trans. on Power Systems, vol. 3, no. 2, pp. 753-762, May 1988.

[10] C. S. Cheng and D. Shirmohammadi, "A three-phase power flow method for real-time distribution system analysis," IEEE Trans on Power Systems, vol. 10, no. 2, pp. 671-769, May 1995.

[11] G. X. Luo and A. Semlyen, "Efficient load flow for large weakly meshed networks," IEEE Trans. on Power Systems, vol. 5, no. 4, pp. 1309-1316, Nov. 1990.

[12] D. Rajicic, R. Ackovski, and R. Taleski, "Voltage correction power flow," IEEE Trans. on Power Delivery, vol. 9, no. 2, pp. 1056-1062, Apr. 1994.

[13] T. H. Chen, M. S. Chen, T. Inoue, P. Kotas, and E. A. Chebli, "Distribution system power flow analysis - A rigid approach," IEEE Trans. on Power Systems, vol. 6, no. 3, pp. 1146-1152, Jul. 1991.

[14] B. K. Chen, M .S. Chen, R. R. Shoults, and C. C. Liang, "Hybrid three phase load flow," IEE Proceedings-C, vol. 137, no. 3, pp. 177-185, May 1990.

[15] Y. Zhu and K. Tomsovic, "Adaptive power flow method for distribution systems with dispersed generation," IEEE Trans. on Power Delivery, vol. 17, no. 3, pp. 822-827, Jul. 2002.

[16] J. R. Carson, "Wave propagation in overhead wires with ground return", Bell System Technical Journal, 1927.

[17] IEEE Distribution Planning Working Group Report, "Radial distribution test feeders," IEEE Trans. on Power Systems, vol. 6, no. 3, pp. 975-985, Aug. 1991.

[18] IEEE Distribution System Analysis Subcommittee Report, "Radial distribution test feeders," in 2000 PES Summer Meeting. Available: http://ewh.ieee.org/soc/pes/dsacom/testfeeders.html

[19] X. Zhang, F. Soudi, D. Shirmohammadi, and C. S. Cheng, "A distribution short circuit analysis approach using hybrid compensation method," IEEE Trans. on Power Systems, vol.10, no. 4, pp. 2053-2059, Nov. 1995.

[20] A. Tan, W. H. Edwin Liu, and D. Shirmohammadi, "Transformer and load modeling in short circuit analysis for distribution systems," IEEE Trans. on Power Systems, vol. 12, no. 3, pp. 1315-1322, Aug. 1997.

\section{BIOGRAPHIES}

Rade M. Ciric graduated from the University of Novi Sad in 1987, received his M.Sc. degree from the University of Belgrade in 1992 and gained his Ph.D. degree from University of Novi Sad, Yugoslavia, in 2000, all in electrical engineering. He was with Electric Power Industry of Serbia Elektrovojvodina from 1987 to 2001. From 1997 to 2001 he was also an associate researcher at Group for DMS at Univ. of Novi Sad. Presently he is a postdoctoral researcher at UNESP - Ilha Solteira, SP, Brazil. His current research interests include all aspects of Distribution Management System. He is a committee member of CIRED-Yugoslavia and DistribuTECH Europe. e-mail: rciric@netscape.net

Antonio Padilha Feltrin (M'89) obtained the B.Sc. degree from EFEI, the M.Sc. and Ph.D. from UNICAMP, Brazil. He is currently an Associate Professor at UNESP - Ilha Solteira, SP, Brazil. From 1995 to 1997 he was a Visiting Faculty at ECE Department of University of Wisconsin - Madison. His main interests are in analysis and control of power systems.

e-mail: padilha@dee.feis.unesp.br

Luis F. Ochoa (S'01) graduated from the National Engineering University (U.N.I.) - Lima, Peru, in 2000. Presently he is a M.Sc. Student in Electrical Power Systems at the UNESP - Ilha Solteira, SP, Brazil. His main interest is distribution system analysis.

e-mail: luis_ochoa@ieee.org 